\title{
CONVERGENCE OF SECOND-ORDER SCHEMES FOR ISENTROPIC GAS DYNAMICS
}

\author{
GUI-QIANG CHEN AND JIAN-GUO LIU
}

\begin{abstract}
Convergence of a second-order shock-capturing scheme for the system of isentropic gas dynamics with $L^{\infty}$ initial data is established by analyzing the entropy dissipation measures. This scheme is modified from the classical MUSCL scheme to treat the vacuum problem in gas fluids and to capture local entropy near shock waves. Convergence of this scheme for the piston problem is also discussed.
\end{abstract}

\section{INTRODUCTION}

We are concerned with the following system of isentropic gas dynamics:

$$
\left\{\begin{array}{l}
\rho_{t}+m_{x}=0, \\
m_{t}+\left(m^{2} / \rho+p(\rho)\right)_{x}=0,
\end{array}\right.
$$

where $\rho$ is the density, $m$ is the momentum, $p(\rho)=\rho^{\gamma} / \gamma$ is the pressure, and $1<\gamma \leq 5 / 3$ is the adiabatic exponent. Because of the presence of vacuum, $\rho=0$, the system is not strictly hyperbolic. This fact causes many difficulties in theoretical analysis and numerical computation. Away from the vacuum, we can define $u=m / \rho$ as the velocity.

We focus mainly on the convergence of high-order difference schemes for the Cauchy problem with $L^{\infty}$ initial data for (1.1):

$$
\left.(\rho, m)\right|_{t=0}=\left(\rho_{0}(x), m_{0}(x)\right),
$$

where $m_{0}(x)$ and $\rho_{0}(x) \geq 0(\not \equiv 0)$ are bounded measurable functions. We also discuss a mixed initial-boundary problem on a domain $D$-the piston problem:

$$
\begin{cases}\left.(\rho, m)\right|_{t=0}=\left(\rho_{0}(x), m_{0}(x)\right), & 0<x<L, \\ m\left(x_{j}(t), t\right)-\rho\left(x_{j}(t), t\right) u_{j}(t)=0, & t>0, j=1,2,\end{cases}
$$

where

$$
\left\{\begin{array}{l}
D=\left\{(x, t): x_{1}(t)<x<x_{2}(t), t>0\right\}, \\
x_{1}(t)=\int_{0}^{t} u_{1}(s) d s, \quad x_{2}(t)=L+\int_{0}^{t} u_{2}(s) d s,
\end{array}\right.
$$

Received by the editor August 9, 1991 and, in revised form, May 28, 1992.

1991 Mathematics Subject Classification. Primary 65M12; Secondary 35L65, 76N15.

Gui-Qiang Chen's research was supported in part by IMA at Minnesota with funds provided by NSF, by ONR grant N00014-91-J-1384, by DOE grant under Contract W-31-109-Eng-38, and by an Alfred P. Sloan Foundation Fellowship. Jian-Guo Liu's research was supported in part by NSF grant DMS-8505550 as a postdoctoral fellow at the MSRI at Berkeley, and by DOE grant DE-FG02-88ER25053 as a visiting member at the Courant Institute. 
$u_{1}(t)$ and $u_{2}(t)$ are bounded measurable functions that denote the velocities of the pistons, and $x_{1}(t)$ and $x_{2}(t)$ denote the moving boundaries.

MUSCL (monotonic upstream centered schemes for conservation laws) schemes are the second-order schemes, first introduced by van Leer [24]. Beginning with the cell average of initial data, one first constructs a linear function on each cell to approximate the initial data. Second, one solves the equation with the piecewise linear function just constructed as initial data for a small time step. Then one averages the solution at each small cell, reconstructs a piecewise linear function again, and repeats these steps again and again. An essential requirement in reconstructing the piecewise linear functions is that they do not contain intolerable oscillations. This technique, called reconstruction, has been generalized to high-order polynomials. The generalized schemes are called Godunov-type schemes (cf. the Godunov scheme [8]). The piecewise parabolic method (PPM) [27] is an example of a third-order scheme, and essentially nonoscillatory (ENO) schemes $[9,10]$ are examples of even higher-order schemes. Like the MUSCL schemes, the Godunov-type schemes can be written in the the following abstract form (cf. [9]):

$$
v^{n+1}=E_{h}(\Delta t) \circ v^{n} \equiv A_{h} \circ E(\Delta t) \circ R\left(\cdot, v^{n}\right) .
$$

Here, $v^{n}$ is the numerical solution approximating $v(\cdot, n \Delta t), A_{h}$ is the cellaveraging operator, $E(t)$ is the exact evolution operator, and $R(x, v)$ is the reconstruction step, where $h$ and $\Delta t$ are the space and time step sizes, respectively. The Godunov-type schemes represent the following "modern techniques" in the numerical computations of hyperbolic systems of conservation laws: achieving high accuracy at least where the solution is smooth, producing good resolution for the shocks and the contact discontinuities, and avoiding superfluous oscillations and nonphysical discontinuities (e.g., expansion shocks).

In applying the MUSCL schemes to the system of isentropic gas dynamics, we need to modify the slopes in the reconstructed piecewise linear functions at points near the vacuum to avoid negative density, which may be produced in the reconstruction step. We also need to modify the slopes at points of steep gradient of the solutions to capture local entropy. Since our analysis is based on a uniform $L^{\infty}$ estimate that is much weaker than the BV (bounded variation) estimate, we can relax the TVD (total variation diminishing) requirements for the reconstruction step of the standard MUSCL schemes. The MUSCL schemes with the above-mentioned slope modification can achieve second-order accuracy in the smooth regions of the solutions. Of course, as is well known, it is impossible to get high-order accuracy near shock waves and the vacuum states. The slope modification technique can be easily implemented numerically. Some similar modification techniques were also applied to high-order schemes for conservation laws (cf. [4, 5, 12, 14, 20, 21, 25, 26]).

For one-dimensional scalar conservation laws, one advantage of TVD schemes or, even more generally, TVB (total variation bounded) schemes, is that there is always a convergent subsequence based on the Helly principle. Thus, the crucial task is to prove that the limit solution satisfies the entropy inequalities. Osher [17] proved the convergence of a class of semidiscrete generalized MUSCL schemes for the strictly convex case. For further discussions, also see $[13,28]$. For systems of conservation laws, it is generally impossible to get TV 
(total variation) estimates for difference schemes, even the TVB schemes.

The main objective of this paper is to prove the convergence of this secondorder shock-capturing scheme. By convergence here we mean that there is a subsequence that converges to a generalized solution satisfying the entropy condition. As long as the entropy solution is unique, the whole sequence converges to the physical solution. In order to get convergence, we need some compactness and entropy inequality estimates for the approximate solutions. More specifically, we estimate a uniform bound for the approximate solutions $v_{h}$ and estimate the $H_{\mathrm{loc}}^{-1}$ compactness for the entropy dissipation measures $\mu_{(\eta, q)}^{h}=\eta\left(v_{h}\right)_{t}+q\left(v_{h}\right)_{x}$ associated with all weak entropy pairs $(\eta, q)$. This method is based on the idea of compensated compactness, which was first studied by Murat and Tartar [15, 23] and successfully applied to the system of isentropic gas dynamics by DiPerna [7] for viscosity approximations for the case $\gamma=1+\frac{2}{2 m+1}, m \geq 2$ integers, and by Chen [2] and by Ding, Chen, and Luo [6] for the Godunov scheme and the Lax-Friedrichs scheme for the general case $1<\gamma \leq 5 / 3$.

We achieve a uniform bound for the approximate solutions $v_{h}$ by combining the principle of invariant regions for systems of conservation laws with some careful estimates, especially near the vacuum points (see $\S 4.1$ ). The main techniques (described briefly below and in detail in $\S 4.2)$ involve $H_{\text {loc }}^{-1}$ compactness estimates of the weak entropy dissipation measures $\eta\left(v_{h}\right)_{t}+q\left(v_{h}\right)_{x}$. We achieve the entropy inequalities by following the techniques from the compactness estimates (see $\S 4.3$ ).

First, by the dominant property of mechanical entropy, we can show that any weak entropy pair is a linear combination of a convex weak entropy pair and the mechanical entropy pair, which is also convex (also see [3]). Thus we need only the compactness estimates for all convex entropy pairs. Next, by duality and the Sobolev interpolation inequality, we need to estimate only the $W_{\text {loc }}^{-1, p}$ compactness and the $W_{\text {loc }}^{-1, q}$ bound of the convex entropy dissipation measures for some $1<p \leq 2<q<\infty$, instead of the $H_{\text {loc }}^{-1}$ compactness [1]. The $W_{\text {loc }}^{-1, q}$ bound estimate of the dissipation measures is a direct consequence of the uniform bound of approximate solutions $v_{h}$.

The dissipation measures are supported mainly on the shocks of the approximate solutions in the regions $R \times\left(t_{n-1}, t_{n}\right)$, and on the interfacial lines $R \times\left\{t_{n}\right\}$ for all discrete time steps $t_{n}$. We refer to them as the shock parts of the dissipation measures and the interfacial parts of the dissipation measures, respectively. On the time intervals $\left(t_{n-1}, t_{n}\right), v_{h}$ are generalized solutions constructed by the Godunov scheme. Hence, by the entropy inequalities of the generalized solutions from the Godunov scheme, we know that the shock parts of the dissipation measures are negative for any convex entropy pair. Since the embedding of the positive cone of $W^{-1, p}$ in $W^{-1, q}$ is completely continuous for all $q<p$ [15], we need only to estimate the $W_{\text {loc }}^{-1, p}$ bound of the interfacial parts of the dissipation measures on the interfacial lines for some $p>1$.

The most detailed calculation, of course, involves bound estimates of the interfacial parts of the dissipation measures, which come from the averaging process and the reconstruction process of the scheme. The averaging process is entropy-decreasing, while the reconstruction process usually is entropyincreasing. We denote the dissipation measures from these processes as $\mathscr{A}_{(\eta, q)}^{h}$ 
and $\mathscr{R}_{(\eta, q)}^{h}$, respectively, and estimate each of them separately. One difficulty in the estimates is the singularity of the Hessian matrices of weak entropies at the vacuum points. To overcome this difficulty, we use an exact mean value formula to expand the errors near the vacuum points. We also use the following properties of the singularity. In the physical plane $\rho-m$, the Hessian matrices of the weak entropies have singularities at the vacuum points, but they are dominated by the Hessian matrix of the mechanical energy. In the Riemann invariants plane $w-z$, however, the Hessian matrix of any weak entropy has no singularity. We estimate the mechanical energy first and estimate the general weak entropies afterwards. We point out that some of these estimates were discussed in [7] in the analysis of the convergence of the viscosity solutions to the system of isentropic gas dynamics, but our estimates for the difference schemes involve more careful analysis and more sophisticated techniques to handle the discrete errors. The estimate of the averaging process is similar to that of the Godunov scheme [2, 6].

We can summarize the above steps as the following strategy to analyze the convergence of Godunov-type schemes for systems of conservation laws. First, we construct global solutions for the Riemann problems by using classical methods; it is easy to check the properties of the Riemann solutions, such as entropy inequalities and invariant regions. Then we show that the approximate solutions of the Godunov scheme for general initial value problems or mixed initial-boundary value problems have subsequences that converge to a generalized solution. We refer to this solution as the generalized solution constructed by the Godunov scheme. We can show that the generalized solution retains many important properties, such as entropy inequalities and invariant regions. Once we have the generalized solutions for the Cauchy problems or the boundary value problems, we can construct and analyze higher-order schemes. For example, the piston problem discussed by Nishida and Smoller [16] are very complicated, mainly as a result of the reflection of shock waves at the rigid wall, where the strength of the reflected shock is usually greater than the strength of the incoming shock. We can easily use this strategy to prove the convergence of the second-order scheme for the piston problem (1.1) and (1.3) (see $\S 5$ for more details). It would be interesting to further analyze the MUSCL scheme and higher-order schemes by using this strategy.

\section{SYSTEM OF ISENTROPIC GAS DYNAMICS}

In this section we first introduce some basic facts about the system of gas dynamics (1.1). For more details, see Chen [1] and Lax [11]. The system (1.1) can be rewritten as follows:

$$
v_{t}+f(v)_{x} \equiv v_{t}+\nabla f(v) v_{x}=0
$$

where

$$
v=\left(\begin{array}{c}
\rho \\
m
\end{array}\right), \quad f(v)=\left(\begin{array}{c}
m \\
m^{2} / \rho+\rho^{\gamma} / \gamma
\end{array}\right)
$$

and

$$
\nabla f=\left(\begin{array}{cc}
0 & 1 \\
-m^{2} / \rho^{2}+\rho^{\gamma-1} & 2 m / \rho
\end{array}\right)
$$


The eigenvalues of the system (1.1) are

$$
\lambda_{1}=\frac{m}{\rho}-\rho^{\theta}, \quad \lambda_{2}=\frac{m}{\rho}+\rho^{\theta},
$$

and the corresponding eigenvectors are

$$
r_{1}=\left(1, \frac{m}{\rho}-\rho^{\theta}\right)^{\top}, \quad r_{2}=\left(1, \frac{m}{\rho}+\rho^{\theta}\right)^{\top},
$$

where $c=\rho^{\theta}$ is the speed of sound and $\theta=(\gamma-1) / 2$. When $\rho=0$, referred to as the vacuum, the two wave speeds coalesce, and the system (1.1) loses strict hyperbolicity, which is an essential feature in gas dynamics and causes many difficulties in theoretical analysis and numerical computation. Define the Riemann invariants

$$
w=\frac{m}{\rho}+\frac{\rho^{\theta}}{\theta}, \quad z=\frac{m}{\rho}-\frac{\rho^{\theta}}{\theta}
$$

which satisfy

$$
\nabla w \cdot r_{1}=\nabla z \cdot r_{2}=0
$$

Denote sets $\Sigma_{C}$ and $\Lambda_{C}$ as

$$
\left\{\begin{array}{l}
\Sigma_{C}=\{(\rho, m): 0 \leq \rho \leq C,|m| \leq C \rho\}, \\
\Lambda_{C}=\{(\rho, m): w \leq C, z \geq-C, w-z \geq 0\} .
\end{array}\right.
$$

Directly from the identities (2.1), we have the following lemma.

Lemma 2.1. For any positive constant $C$, the sets $\Sigma_{C}$ and $\Lambda_{C}$ are closed convex sets in the $\rho-m$ plane. Furthermore, there are positive constants $C_{1}$ and $C_{2}$ such that

$$
\Lambda_{C} \subset \Sigma_{C_{1}} \quad \text { and } \quad \Sigma_{C} \subset \Lambda_{C_{2}} \text {. }
$$

Definition 2.1. A pair of mappings $\eta: R^{2} \rightarrow R, q: R^{2} \rightarrow R$ is called an entropyentropy flux pair if it satisfies the identity

$$
\nabla q=\nabla \eta \nabla f .
$$

Denote $\tilde{\eta}(\rho, m / \rho)=\eta(\rho, m)$. If $\tilde{\eta}(0, u) \equiv 0$, then $\eta$ is called a weak entropy.

Among all entropies, the most natural entropy, the mechanical energy,

$$
\eta_{*}(\rho, m)=\frac{1}{2} \frac{m^{2}}{\rho}+\frac{1}{\gamma(\gamma-1)} \rho^{\gamma},
$$

will play an important role in our analysis. One can easily check that the mechanical energy is a strictly convex weak entropy.

Definition 2.2. We call a functional $\mu_{(\eta, q)}$ an entropy dissipation functional associated with a weak entropy pair $(\eta, q)$ if

$$
\left\langle\mu_{(\eta, q)}, \phi\right\rangle=-\iint_{R_{+}^{2}}\left(\eta(v) \phi_{t}+q(v) \phi_{x}\right) d x d t-\left.\int_{-\infty}^{\infty} \eta(v) \phi\right|_{t=0} d x,
$$

for any function $\phi \in C_{0}^{\infty}\left(R_{+}^{2}\right), R_{+}^{2} \equiv(-\infty, \infty) \times[0, \infty)$. We also denote $\mu_{(\eta, q)}$ as

$$
\mu_{(\eta, q)}=\eta(v)_{t}+q(v)_{x}
$$


If $v$ is replaced by an approximate solution $v_{h}$, then the entropy dissipation functional is denoted as $\mu_{(\eta, q)}^{h}$ and is referred to as an entropy dissipation functional associated with the weak entropy pair $(\eta, q)$ and the approximate solution $v_{h}$.

Remark 2.1. If the function $v$ in (2.6) is a $\mathrm{BV}$ function, then the entropy dissipation functional $\mu_{(\eta, q)}$ is a Radon measure which is referred to as an entropy dissipation measure. The approximate solutions $v_{h}$ constructed in $\S 3$ are BV functions. Therefore, we use the word measure instead of functional throughout this paper.

Definition 2.3. A bounded measurable function $v(x, t)=(\rho(x, t), m(x, t))$ is called a generalized solution of the Cauchy problem (1.1)-(1.2) if it satisfies the following conditions:

(1) For any $\phi(x, t) \in C_{0}^{\infty}\left(R_{+}^{2}\right)$, we have

$$
\iint_{R_{+}^{2}}\left(v \phi_{t}+f(v) \phi_{x}\right) d x d t+\int_{-\infty}^{\infty} v_{0}(x) \phi(x, 0) d x=0 .
$$

(2) For any convex weak entropy pair $(\eta, q)$, and any $\phi(x, t) \in C_{0}^{\infty}\left(R_{+}^{2}\right)$, $\phi \geq 0$ and $\phi(x, 0)=0$, we have

$$
\iint_{R_{+}^{2}}\left(\eta(v) \phi_{t}+q(v) \phi_{x}\right) d x d t \geq 0 .
$$

Remark 2.2. An equivalent definition of the generalized solution to the Cauchy problem (1.1)-(1.2) is that the entropy dissipation measures $\mu_{(\eta, q)}$ associated with all convex weak entropy pairs $(\eta, q)$ are negative.

The following compactness framework and existence theorem of the global generalized solution to the Cauchy problem (1.1)-(1.2) are established in Chen [2] and Ding, Chen, and Luo [6].

Theorem 2.1. Suppose that the approximate solutions $v_{h}(x, t)=\left(\rho_{h}(x, t)\right.$, $\left.m_{h}(x, t)\right)$ for the Cauchy problem (1.1)-(1.2) satisfy the following framework:

(1) There is a constant $C>0$ such that

$$
0 \leq \rho_{h}(x, t) \leq C, \quad\left|m_{h}(x, t)\right| \leq C \rho_{h}(x, t), \quad \text { a.e. }
$$

(2) For any weak entropy pair $(\eta, q)$, the associated entropy dissipation measure

$$
\mu_{(\eta, q)}^{h}=\eta\left(v_{h}\right)_{t}+q\left(v_{h}\right)_{x} \quad \text { is compact in } H_{\mathrm{loc}}^{-1}\left(R_{+}^{2}\right) .
$$

Then there exists a subsequence (still labeled) $\left(\rho_{h}, m_{h}\right)$ such that

$$
\left(\rho_{h}(x, t), m_{h}(x, t)\right) \rightarrow(\rho(x, t), m(x, t)), \quad \text { a.e. }
$$

Theorem 2.2. Suppose that the initial data $v_{0}(x)=\left(\rho_{0}(x), m_{0}(x)\right)$ satisfy

$$
0 \leq \rho_{0}(x) \leq M, \quad\left|m_{0}(x)\right| \leq M \rho_{0}(x),
$$

for a constant $M>0$. Then there exists a global generalized solution $v(x, t)=$ $(\rho(x, t), m(x, t))$ for the Cauchy problem (1.1)-(1.2). In particular, for any 
$t_{1}, t_{2} \in[0, T]$, any convex weak entropy $(\eta, q)$, and any $\phi \in C_{0}^{\infty}\left(R_{+}^{2}\right), \phi \geq 0$, the solution $v(x, t)$ satisfies

$$
\begin{aligned}
& \int_{t_{1}}^{t_{2}} \int_{-\infty}^{\infty}\left(\eta(v) \phi_{t}+q(v) \phi_{x}\right) d x d t \\
& \quad \geq\left.\int_{-\infty}^{\infty} \eta(v) \phi\right|_{t=t_{1}} d x-\left.\int_{-\infty}^{\infty} \eta(v) \phi\right|_{t=t_{2}} d x
\end{aligned}
$$

\section{THE SCHEME}

Let $h$ and $\Delta t$ be the space and time step sizes, respectively. Assume that $h=\lambda \Delta t$, for some appropriate constant $\lambda$ so that the Courant-Friedrichs-Levy condition

$$
\max _{j=1,2}\left|\lambda_{j}(v)\right|<\lambda
$$

holds. Partition the real line $t_{n}=n \Delta t$ into cells, with the $j$ th cell centered at $x_{j}=j h, j=0, \pm 1, \pm 2, \ldots$. Denote $v_{j}^{n}$ as the numerical approximation to the exact solution $v\left(x_{j}, t_{n}\right)$, or the cell average of the exact solution $v\left(x, t_{n}\right)$ on the $j$ th cell $\left(x_{j-1 / 2}, x_{j+1 / 2}\right), x_{j+1 / 2}=(j+1 / 2) h$. Let $\left(w_{j}^{n}, z_{j}^{n}\right)$ be the Riemann invariants of $\left(\rho_{j}^{n}, m_{j}^{n}\right)$, and $\Delta_{+} w_{j}^{n} \equiv w_{j+1}^{n}-w_{j}^{n}, \Delta_{-} w_{j}^{n} \equiv w_{j}^{n}-w_{j-1}^{n}$, etc. Our second-order shock-capturing scheme can be written in the abstract form (1.5). The detailed construction is given below.

Reconstruction. On the cell $\left(x_{j-1 / 2}, x_{j+1 / 2}\right)$, we use the following linear vector function $\left(\rho_{+}^{n}(x), m_{+}^{n}(x)\right)$ to replace the cell average $\left(\rho_{j}^{n}, m_{j}^{n}\right)$ in order to improve the approximation accuracy:

$$
\left(\begin{array}{c}
\rho_{+}^{n}(x) \\
m_{+}^{n}(x)
\end{array}\right)=\left(\begin{array}{c}
\rho_{j}^{n} \\
m_{j}^{n}
\end{array}\right)+\left(\begin{array}{c}
\alpha_{j}^{n} \\
\beta_{j}^{n}
\end{array}\right)\left(x-x_{j}\right),
$$

where $\left(\alpha_{j}^{n}, \beta_{j}^{n}\right)$ is the constant vector given by

$$
\left(\begin{array}{c}
\alpha_{j}^{n} \\
\beta_{j}^{n}
\end{array}\right)=\frac{\left(\rho_{j}^{n}\right)^{1-\theta}}{2}\left(\begin{array}{cc}
1 & -1 \\
\frac{m_{j}^{n}}{\rho_{j}^{n}}+\left(\rho_{j}^{n}\right)^{\theta} & -\frac{m_{j}^{n}}{\rho_{j}^{n}}+\left(\rho_{j}^{n}\right)^{\theta}
\end{array}\right)\left(\begin{array}{c}
s_{j}^{n} \\
t_{j}^{n}
\end{array}\right),
$$

and the constant vector $\left(s_{j}^{n}, t_{j}^{n}\right)$ satisfies the following two conditions:

$$
-M_{0} h \leq \frac{h s_{j}^{n}}{\Delta_{+} w_{j}^{n}}, \frac{h s_{j}^{n}}{\Delta_{-} w_{j}^{n}}, \frac{h t_{j}^{n}}{\Delta_{+} z_{j}^{n}}, \frac{h t_{j}^{n}}{\Delta_{-} z_{j}^{n}} \leq 1+M_{0} h,
$$

where $M_{0} \geq 0$ is a given constant;

(2)

$$
\left|s_{j}^{n}\right|,\left|t_{j}^{n}\right| \leq \min \left(1, w_{j}^{n}-z_{j}^{n}\right) h^{-\alpha},
$$

where $0<\alpha<1 / 2$ is a given constant.

Solution in small time. As a result of the convergence of the Godunov scheme, we can exactly solve the following Cauchy problem for $t_{n} \leq t<t_{n+1}$ :

$$
\left\{\begin{array}{l}
\frac{\partial v_{h}}{\partial t}+\frac{\partial f\left(v_{h}\right)}{\partial x}=0 \\
\left.v_{h}\right|_{t=t_{n}}=v_{+}^{n}(x)
\end{array}\right.
$$

We denote $v_{-}^{n+1}(x)=v_{h}\left(x, t_{n+1}-0\right)$. 

is,

Cell averaging. We take the average of $v_{-}^{n+1}(x)$ in the $j$ th cell as $v_{j}^{n+1}$, that

$$
v_{j}^{n+1}=\frac{1}{h} \int_{x_{j-1 / 2}}^{x_{j+1 / 2}} v_{-}^{n+1}(x) d x .
$$

We also refer to $v_{h}(x, t)=\left(\rho_{h}(x, t), m_{h}(x, t)\right)$ in the above construction steps as the approximate solutions of the second-order scheme.

Remark 3.1. There are many approaches for constructing the slopes $\left(s_{j}^{n}, t_{j}^{n}\right)$ to achieve the condition (3.3). The minmod choice [24], the second-order ENO scheme [10], and the UNO scheme [9] are examples that have been widely used. The minmod choice is TVD and has the following formula:

$$
\left(s_{j}^{n}, t_{j}^{n}\right)=\operatorname{minmod}\left(v_{j+1}^{n}-v_{j}^{n}, v_{j}^{n}-v_{j-1}^{n}\right),
$$

where

$$
\min \bmod (x, y)= \begin{cases}x, & \text { if }|x|<|y|, \\ 0, & \text { if } x y<0, \\ y, & \text { if }|y|<|x| .\end{cases}
$$

Remark 3.2. The condition (3.4) is to prevent negative density near the vacuum points and to capture local entropy near the shock waves. To achieve this condition, we have the following filter (still denoted as $\left.\left(s_{j}^{n}, t_{j}^{n}\right)\right)$ :

$$
\left(s_{j}^{n}, t_{j}^{n}\right) \rightarrow \text { filter }\left(\left(s_{j}^{n}, t_{j}^{n}\right), c_{j}^{n}\right),
$$

where

$$
c_{j}^{n}=\min \left(1, w_{j}^{n}-z_{j}^{n}\right) h^{-\alpha},
$$

and

$$
\text { filter }(x, c)= \begin{cases}x, & \text { if }|x| \leq c, \\ -c, & \text { if } x<-c, \\ c, & \text { if } x>c\end{cases}
$$

We can improve the condition (3.4) near the vacuum to

$$
\left|s_{j}^{n}-t_{j}^{n}\right| \leq\left(w_{j}^{n}-z_{j}^{n}\right) / h
$$

if we reconstruct the piecewise linear functions in the Riemann invariants. In this case, the scheme is no longer conservative in the sense of Lax-Wendroff [12], and we can still prove convergence of this scheme by some modification of the proof in $\S 4$ and $\S 5$.

Remark 3.3. In the "solution in small time" step, we use an exact solution for analytical convenience. In numerical computations, one usually uses numerical approximation solvers such as the Roe solver [18], the Runge-Kutta time discrete technique [19], and the Cauchy-Kowalewski analytic expansion [10], which have been developed to achieve high accuracy and easy implementation.

Remark 3.4. The condition (3.3) is a relaxed BV version of the original MUSCL scheme, in which one takes $M_{0}$ to be zero in (3.3) and the second-order accuracy at the extreme points is lost. The slopes constructed in Remark 3.2, such as the 
minmod, ENO, and UNO choices with the filter (3.7), can achieve the accuracy condition:

$$
\left\{\begin{array}{c}
h s_{j}^{n} / \Delta_{+} w_{j}^{n}=1+O(h)=h s_{j}^{n} / \Delta_{-} w_{j}^{n}, \\
h t_{j}^{n} / \Delta_{+} z_{j}^{n}=1+O(h)=h t_{j}^{n} / \Delta_{-} z_{j}^{n} .
\end{array}\right.
$$

This implies second-order accuracy in the smooth regions (see Osher [17]).

\section{Convergence ANAlysis}

\subsection{Uniform bound.}

Lemma 4.1. Let $\left(w_{+}^{n}(x), z_{+}^{n}(x)\right)$ be the Riemann invariants of $\left(\rho_{+}^{n}(x), m_{+}^{n}(x)\right)$. Suppose that there is a constant $C_{n}>0$ such that $\left(\rho_{j}^{n}, m_{j}^{n}\right) \in \Lambda_{C_{n}}$ for all integers $j$. Then we have

$$
\left\{\begin{array}{c}
\left|w_{+}^{n}(x)-w_{j}^{n}-s_{j}^{n}\left(x-x_{j}\right)\right| \leq \frac{40}{\theta^{2}} h^{2-2 \alpha} C_{n}, \\
\left|z_{+}^{n}(x)-z_{j}^{n}-t_{j}^{n}\left(x-x_{j}\right)\right| \leq \frac{40}{\theta^{2}} h^{2-2 \alpha} C_{n},
\end{array}\right.
$$

as $h^{1-\alpha} \leq \theta / 2$, where $\theta=(\gamma-1) / 2$ and $0<\alpha<1 / 2$.

Proof. Directly from (3.2) and (3.4), we have

$$
\left|\alpha_{j}^{n}\right|=\frac{1}{2}\left(\rho_{j}^{n}\right)^{1-\theta}\left|s_{j}^{n}-t_{j}^{n}\right| \leq\left(\rho_{j}^{n}\right)^{1-\theta}\left(w_{j}^{n}-z_{j}^{n}\right) h^{-\alpha}=\frac{2}{\theta} \rho_{j}^{n} h^{-\alpha},
$$

and

$$
\begin{aligned}
\left|\beta_{j}^{n}\right| & =\frac{\left(\rho_{j}^{n}\right)^{1-\theta}}{2}\left|\frac{m_{j}^{n}}{\rho_{j}^{n}}\left(s_{j}^{n}-t_{j}^{n}\right)+\left(\rho_{j}^{n}\right)^{\theta}\left(s_{j}^{n}+t_{j}^{n}\right)\right| \\
& =\frac{\left(\rho_{j}^{n}\right)^{1-\theta}}{4}\left|\left(w_{j}^{n}+z_{j}^{n}\right)\left(s_{j}^{n}-t_{j}^{n}\right)+\theta\left(w_{j}^{n}-z_{j}^{n}\right)\left(s_{j}^{n}+t_{j}^{n}\right)\right| \\
& \leq C_{n}\left(\rho_{j}^{n}\right)^{1-\theta}\left(w_{j}^{n}-z_{j}^{n}\right) h^{-\alpha}=\frac{2}{\theta} \rho_{j}^{n} h^{-\alpha} C_{n} .
\end{aligned}
$$

The estimates (4.2) and (4.3) together with (3.1) lead to

$$
\left\{\begin{aligned}
&\left|\rho_{+}^{n}(x)-\rho_{j}^{n}\right| \leq \frac{1}{\theta} \rho_{j}^{n} h^{1-\alpha}, \\
&\left|m_{+}^{n}(x)-m_{j}^{n}\right| \leq \frac{1}{\theta} \rho_{j}^{n} h^{1-\alpha} C_{n} .
\end{aligned}\right.
$$

Taking the Taylor expansion for $w_{+}^{n}$ and $z_{+}^{n}$ gives

$$
\left\{\begin{array}{c}
w_{+}^{n}(x)=w_{j}^{n}+s_{j}^{n}\left(x-x_{j}\right)+a_{j}^{n}\left(x-x_{j}\right)^{2}, \\
z_{+}^{n}(x)=z_{j}^{n}+t_{j}^{n}\left(x-x_{j}\right)+b_{j}^{n}\left(x-x_{j}\right)^{2},
\end{array}\right.
$$

where

$$
\left\{\begin{array}{l}
a_{j}^{n}=\left(2 \tilde{m}_{j}^{n} / \tilde{\rho}_{j}^{n}+(\theta-1)\left(\tilde{\rho}_{j}^{n}\right)^{\theta}\right) \frac{\left(\alpha_{j}^{n}\right)^{2}}{\left(\tilde{\rho}_{j}^{n}\right)^{2}}-2 \frac{\alpha_{j}^{n} \beta_{j}^{n}}{\left(\tilde{\rho}_{j}^{n}\right)^{2}} \\
b_{j}^{n}=\left(2 \tilde{m}_{j}^{n} / \tilde{\rho}_{j}^{n}-(\theta-1)\left(\tilde{\rho}_{j}^{n}\right)^{\theta}\right) \frac{\left(\alpha_{j}^{n}\right)^{2}}{\left(\tilde{\rho}_{j}^{n}\right)^{2}}-2 \frac{\alpha_{j}^{n} \beta_{j}^{n}}{\left(\tilde{\rho}_{j}^{n}\right)^{2}},
\end{array}\right.
$$


and $\tilde{\rho}_{j}^{n}$ and $\tilde{m}_{j}^{n}$ are the mean values. We have from (4.4) that

$$
\frac{1}{\left|\tilde{\rho}_{j}^{n}\right|} \leq \frac{1}{\rho_{j}^{n}} \frac{\theta}{\theta-h^{1-\alpha}} \leq \frac{1}{\rho_{j}^{n}}\left(1+\frac{2}{\theta} h^{1-\alpha}\right) \leq \frac{2}{\rho_{j}^{n}},
$$

and

$$
\frac{\tilde{m}_{j}^{n}}{\tilde{\rho}_{j}^{n}} \leq 2\left(\frac{\left|m_{j}^{n}\right|}{\rho_{j}^{n}}+\frac{1}{\theta} h^{1-\alpha} C_{n}\right) \leq 3 C_{n},
$$

as $h^{1-\alpha} \leq \theta / 2$. Since $0<\theta \leq 1$, one has

$$
\left(\tilde{\rho}_{j}^{n}\right)^{\theta} \leq\left(2 \rho_{j}^{n}\right)^{\theta}=\frac{1}{2} 2^{\theta} \theta\left(w_{j}^{n}-z_{j}^{n}\right) \leq 2 C_{n} .
$$

Using (4.2)-(4.3) and (4.6)-(4.8), one obtains

$$
\left|a_{j}\right|,\left|b_{j}\right| \leq \frac{160}{\theta^{2}} h^{-2 \alpha} C_{n} .
$$

Therefore,

$$
\left\{\begin{array}{l}
\left|a_{j}^{n}\left(x-x_{j}\right)^{2}\right| \leq \frac{40}{\theta^{2}} h^{2-2 \alpha} C_{n}, \\
\left|b_{j}^{n}\left(x-x_{j}\right)^{2}\right| \leq \frac{40}{\theta^{2}} h^{2-2 \alpha} C_{n} .
\end{array}\right.
$$

Combining (4.5) and (4.9) gives (4.1).

The property of the invariant regions for the Riemann problems can be found in Chen [1]. As a direct consequence of the convergence of the Godunov scheme, we know that the generalized solutions constructed by the Godunov scheme for the Cauchy problem also have invariant regions. This gives the following lemma.

Lemma 4.2. The regions $\Lambda_{C}$ are invariant regions for the Cauchy problem (1.1)(1.2). More precisely, if the initial data belong to $\Lambda_{C}$, then the generalized solutions constructed by the Godunov scheme for the Cauchy problem also belong to $\Lambda_{C}$.

Theorem 4.1. Let $v_{h}=\left(\rho_{h}, m_{h}\right)$ be the approximate solutions of the secondorder scheme (1.5). Assume the initial data $v_{j}^{0} \in \Sigma_{M}$ for some constant $M>0$. Then there is a constant $M_{T}>0$ independent of $h$ such that $v_{h}(x, t) \in \Lambda_{M_{T}}$ for all $x \in R, 0<t \leq T$.

Proof. Lemma 2.1 tells us that there is a constant $C_{0}>0$ such that

$$
v_{j}^{0}=\left(\rho_{j}^{0}, m_{j}^{0}\right) \in \Sigma_{M} \subset \Lambda_{C_{0}} .
$$

Lemma 4.1 implies

$$
v_{+}^{0}(x) \in \Lambda_{C_{0}\left(1+N_{0} h\right)}
$$

and, for any $x \in R, 0 \leq t<t_{1}=\Delta t$,

$$
v_{h}(x, t) \in \Lambda_{C_{0}\left(1+N_{0} h\right)} .
$$

Using Lemma 2.1 , one knows that $\Lambda_{C_{0}\left(1+N_{0} h\right)}$ is a convex set in the $\rho-m$ plane. Thus,

$$
v_{j}^{1}=\frac{1}{h} \int_{x_{j-1 / 2}}^{x_{j+1 / 2}} v_{-}^{1}(x) d x \in \Lambda_{C_{0}\left(1+N_{0} h\right)} .
$$


Repeating these arguments gives

$$
v_{h}(x, t) \in \Lambda_{C_{0}\left(1+N_{0} h\right)^{n}} \subset \Lambda_{C_{0} \exp \left(N_{0} T\right)},
$$

for any $x \in R, 0 \leq t<t_{n} \leq T$. Using Lemma 2.1 again, we obtain

$$
v_{h}(x, t) \in \Lambda_{C_{0} \exp \left(N_{0} T\right)} \subset \Sigma_{M_{T}},
$$

for any $x \in R, 0 \leq t \leq T$. This completes the proof of the theorem.

4.2. Compactness of the entropy dissipation measures. In this subsection we estimate the $H_{\text {loc }}^{-1}$ compactness of the entropy dissipation measures $\mu_{(\eta, q)}^{h}=$ $\eta\left(v_{h}\right)_{t}+q\left(v_{h}\right)_{x}$ associated with all weak entropy pairs $(\eta, q)$ and the approximate solutions $v_{h}$ of the second-order scheme. We first estimate the entropy dissipation measures resulting from the reconstruction process and the averaging process of the scheme. The following definition describes each of them.

Definition 4.1. We call Radon measures $\mathscr{R}_{(\eta, q)}^{h}$ and $\mathscr{A}_{(\eta, q)}^{h}$ the dissipation measures resulting from the reconstruction process and the averaging process of the dissipation measure $\mu_{(\eta, q)}^{h}$, respectively, if for any function $\phi \in C_{0}^{\infty}\left(R_{+}^{2}\right)$,

$$
\left\{\begin{array}{l}
\left\langle\mathscr{R}_{(\eta, q)}^{h}, \phi\right\rangle=\sum_{j, n} \int_{x_{j-1 / 2}}^{x_{j+1 / 2}}\left(\eta\left(v_{+}^{n}\right)-\eta\left(v_{j}^{n}\right)\right) \phi\left(x, t_{n}\right) d x, \\
\left\langle\mathscr{A}_{(\eta, q)}^{h}, \phi\right\rangle=\sum_{j, n} \int_{x_{j-1 / 2}}^{x_{j+1 / 2}}\left(\eta\left(v_{j}^{n}\right)-\eta\left(v_{-}^{n}\right)\right) \phi\left(x, t_{n}\right) d x .
\end{array}\right.
$$

The construction of the scheme (specifically, the solution in small time step) says that $v_{h}(x, t)$ is a generalized solution for the Cauchy problem (1.1)-(1.2) on the time interval $\left(t_{n-1}, t_{n}\right)$. For any convex weak entropy pair $(\eta, q)$ and any positive function $\phi \in C_{0}\left(R_{+}^{2}\right)$, Theorem 2.1 states that

$$
\begin{aligned}
& \int_{t_{n-1}}^{t_{n}} \int_{-\infty}^{\infty}\left(\eta\left(v_{h}\right) \phi_{t}+q\left(v_{h}\right) \phi_{x}\right) d x d t \\
& \quad \geq \int_{-\infty}^{\infty} \eta\left(v_{-}^{n}\right) \phi\left(x, t_{n}\right) d x-\int_{-\infty}^{\infty} \eta\left(v_{+}^{n-1}\right) \phi\left(x, t_{n-1}\right) d x
\end{aligned}
$$

Summing over all time intervals, we have

$$
\begin{gathered}
\iint_{R_{+}^{2}}\left(\eta\left(v_{h}\right) \phi_{t}+q\left(v_{h}\right) \phi_{x}\right) d x d t+\int_{-\infty}^{\infty} \eta\left(v_{+}^{0}\right) \phi(x, 0) d x \\
\geq \sum_{n=1}^{\infty} \int_{-\infty}^{\infty}\left(\eta\left(v_{-}^{n}\right)-\eta\left(v_{+}^{n}\right)\right) \phi\left(x, t_{n}\right) d x,
\end{gathered}
$$

or

$$
\left\langle\mu_{(\eta, q)}^{h}, \phi\right\rangle \leq \sum_{n=1}^{\infty} \int_{-\infty}^{\infty}\left(\eta\left(v_{+}^{n}\right)-\eta\left(v_{-}^{n}\right)\right) \phi\left(x, t_{n}\right) d x .
$$

The right side of this inequality is the interfacial part of the dissipation measure $\mu_{(\eta, q)}^{h}$, which can be decomposed into the sum of the dissipation measures resulting from the reconstruction process and the averaging process $(4.10)$. Thus,

$$
\mu_{(\eta, q)}^{h} \leq \mathscr{R}_{(\eta, q)}^{h}+\mathscr{A}_{(\eta, q)}^{h} .
$$


If we take $(v, f(v))$ as the entropy pair in (4.11), then the inequality (4.12) becomes an equality because of the Rankine-Hugoniot condition, namely,

$$
\mu_{(v, f(v))}^{h}=\mathscr{R}_{(v, f(v))}^{h}+\mathscr{A}_{(v, f(v))}^{h} .
$$

One difficulty in estimating dissipation measures is due to the singularity of the Hessian matrices of the weak entropies at the vacuum points. The following lemma describes the properties of the singularity. In the physical plane $\rho-m$, the Hessian matrices of weak entropies have singularities at the vacuum points; however, they are dominated by the Hessian matrix of the mechanical energy. But, in the Riemann invariant plane $w-z$, the Hessian matrix of any weak entropy has no singularity.

Lemma 4.3. Let $(\eta, q)$ be any weak entropy pair, and let $\left(\eta_{*}, q_{*}\right)$ be the mechanical energy pair. Then, on the set $\Sigma_{K}$, we have that

$$
\left|\nabla_{(w, z)} \eta\right| \leq C, \quad\left|\nabla_{(w, z)}^{2} \eta\right| \leq C,
$$

and, for any vectors $r_{i}$ and $r_{j}$,

$$
\left|\nabla_{(\rho, m)} \eta\right| \leq C, \quad\left|r_{i}^{\top} \cdot \nabla_{(\rho, m)}^{2} \eta \cdot r_{j}\right| \leq C r_{i}^{\top} \cdot \nabla_{(\rho, m)}^{2} \eta_{*} \cdot r_{j},
$$

where $C$ is a constant depending only on $\eta$ and the constant $K>0$. We use the same notation $\eta$ in the $\rho-m$ plane and the $w-z$ plane in the subsequent development.

Proof. We conclude from the entropy equation (2.3) that any weak entropy $\eta$ satisfies the following Euler-Poisson-Darboux equation:

$$
\eta_{w z}+\frac{\beta}{w-z}\left(\eta_{w}-\eta_{z}\right)=0,
$$

where $\beta=\frac{3-\gamma}{2(\gamma-1)}>0$. Solving this equation, we know that any weak entropy $\eta$ has the Darboux representation formula

$$
\eta(w, z)=\int_{z}^{w}[(w-s)(s-z)]^{\beta} \phi(s) d s
$$

for any continuous function $\phi(s)$. By changing the variables, we have

$$
\eta(w, z)=(w-z)^{2 /(\gamma-1)} \int_{0}^{1}[\tau(1-\tau)]^{\beta} \phi(w-(w-z) \tau) d \tau .
$$

A possible singularity for $\eta$ can occur only at the vacuum points. Since the leading term is $(w-z)^{2 /(\gamma-1)}, 1<\gamma \leq 5 / 3$, we know that the gradient and the Hessian matrix of the weak entropy have no singularity in the plane $w-z$. This fact gives the estimate (4.15). The estimates (4.16) can be found in [1].

We assume that the initial data $v_{0}(x)=\left(\rho_{0}(x), m_{0}(x)\right) \in \Sigma_{M}$, for some constant $M>0$. We know from Theorem 4.1 that there is a constant $M_{T}>0$ independent of $h$ such that $v_{h}(x, t) \in \Sigma_{M_{T}}$ for any $(x, t) \in R \times[0, T]$. Therefore, the assumption of Lemma 4.3 is valid for $v_{h}$. 
Lemma 4.4. Let $v_{h}=\left(\rho_{h}, m_{h}\right)$ be the approximate solutions of the scheme (1.5), $\eta$ be a weak entropy, and let $\Omega \subset R_{+}^{2}$ be a bounded open set. Then we have

$$
\left\{\begin{array}{l}
\sum_{\left(x_{j}, t_{n}\right) \in \Omega}\left|\int_{x_{j-1 / 2}}^{x_{j+1 / 2}}\left(\eta\left(v_{+}^{n}\right)-\eta\left(v_{j}^{n}\right)\right) d x\right| \leq C h^{1-2 \alpha}, \\
\sum_{\left(x_{j}, t_{n}\right) \in \Omega} \int_{x_{j-1 / 2}}^{x_{j+1 / 2}}\left|v_{+}^{n}-v_{j}^{n}\right|^{2} d x \leq C h^{1-2 \alpha},
\end{array}\right.
$$

where $C$ is a positive constant independent of $h$.

Proof. Since $w_{+}^{n}(x)$ and $z_{+}^{n}(x)$ are the Riemann invariants of $v_{+}^{n}(x)=\left(\rho_{+}^{n}(x)\right.$, $\left.m_{+}^{n}(x)\right)$, we have

$$
\int_{x_{j-1 / 2}}^{x_{j+1 / 2}}\left(\eta\left(v_{+}^{n}\right)-\eta\left(v_{j}^{n}\right)\right) d x=\int_{x_{j-1 / 2}}^{x_{j+1 / 2}}\left(\eta\left(w_{+}^{n}, z_{+}^{n}\right)-\eta\left(w_{j}^{n}, z_{j}^{n}\right)\right) d x,
$$

where we use the same notation $\eta$ in the $\rho-m$ plane and the $w-z$ plane.

On the cell $\left(x_{j-1 / 2}, x_{j+1 / 2}\right)$, taking the Taylor expansion for $\eta\left(w_{+}^{n}, z_{+}^{n}\right)$ leads to

$$
\begin{aligned}
\eta\left(w_{+}^{n},\right. & \left.z_{+}^{n}\right)-\eta\left(w_{j}^{n}, z_{j}^{n}\right) \\
= & \nabla_{(w, z)} \eta\left(w_{j}^{n}, z_{j}^{n}\right)\left(w_{+}^{n}-w_{j}^{n}, z_{+}^{n}-z_{j}^{n}\right)^{\top} \\
& +\frac{1}{2}\left(w_{+}^{n}-w_{j}^{n}, z_{+}^{n}-z_{j}^{n}\right) \nabla_{(w, z)}^{2} \eta(\xi)\left(w_{+}^{n}-w_{j}^{n}, z_{+}^{n}-z_{j}^{n}\right)^{\top},
\end{aligned}
$$

where $\xi$ is a vector intermediate between $\left(w_{+}^{n}, z_{+}^{n}\right)$ and $\left(w_{j}^{n}, z_{j}^{n}\right)$. Integrating the first term on the right-hand side of (4.18) on the cell $\left(x_{j-1 / 2}, x_{j+1 / 2}\right)$ and using (4.1), one has

$$
\begin{aligned}
& \left|\int_{x_{j-1 / 2}}^{x_{j+1 / 2}} \nabla_{(w, z)} \eta\left(w_{j}, z_{j}\right)\left(w_{+}^{n}-w_{j}^{n}, z_{+}^{n}-z_{j}^{n}\right)^{\top} d x\right| \\
& \quad \leq C\left|\int_{x_{j-1 / 2}}^{x_{j+1 / 2}}\left(s_{j}^{n}\left(x-x_{j}\right)+h^{2-2 \alpha}\right) d x\right| \leq C h^{3-2 \alpha} .
\end{aligned}
$$

The bound of $v_{h}$ and Lemma 4.3 implies

$$
\begin{aligned}
& \left|\left(w_{+}^{n}-w_{j}^{n}, z_{+}^{n}-z_{j}^{n}\right) \nabla_{(w, z)}^{2} \eta(\xi)\left(w_{+}^{n}-w_{j}^{n}, z_{+}^{n}-z_{j}^{n}\right)^{\top}\right| \\
& \quad \leq C\left|\left(w_{+}^{n}-w_{j}^{n}, z_{+}^{n}-z_{j}^{n}\right)\right|^{2} \leq C h^{2-2 \alpha} .
\end{aligned}
$$

The last inequality comes from (3.4) in the reconstruction step of our scheme and (4.1). This fact gives us

$$
\left|\int_{x_{j-1 / 2}}^{x_{j+1 / 2}}\left(\eta\left(w_{+}^{n}, z_{+}^{n}\right)-\eta\left(w_{j}^{n}, z_{j}^{n}\right)\right) d x\right| \leq C h^{3-2 \alpha} .
$$

Summing over all cells leads to

$$
\sum_{\left(x_{j}, t_{n}\right) \in \Omega}\left|\int_{x_{j-1 / 2}}^{x_{j+1 / 2}}\left(\eta\left(w_{+}^{n}, z_{+}^{n}\right)-\eta\left(w_{j}^{n}, z_{j}^{n}\right)\right) d x\right| \leq C \sum_{\left(x_{j}, t_{n}\right) \in \Omega} h^{3-2 \alpha} \leq C h^{1-2 \alpha} .
$$


Since the mapping of $(\rho, m)$ to the Riemann invariants $(w, z)$ is a $C^{1}$ mapping, one obtains

$$
\int_{x_{j-1 / 2}}^{x_{j+1 / 2}}\left|v_{+}^{n}-v_{j}^{n}\right|^{2} d x \leq C \int_{x_{j-1 / 2}}^{x_{j+1 / 2}}\left|\left(w_{+}^{n}-w_{j}^{n}, z_{+}^{n}-z_{j}^{n}\right)\right|^{2} d x .
$$

The second part of inequalities (4.17) comes directly from (4.19) and (4.20). This completes the proof of the lemma.

Lemma 4.5. Let $\mathscr{R}_{(\eta, q)}^{h}$ be the dissipation measure resulting from the reconstruction process, and let $\Omega \subset R_{+}^{2}$ be a bounded open set. Then, for any weak entropy pair $(\eta, q)$, we have

$$
\left\|\mathscr{R}_{(\eta, q)}^{h}\right\|_{\left(C_{0}^{1 / 2}(\Omega)\right)^{*}} \leq C h^{1 / 2-\alpha},
$$

where $\left(C_{0}^{1 / 2}(\Omega)\right)^{*}$ is the dual space of $C_{0}^{1 / 2}(\Omega)$.

Proof. For any $\phi \in C_{0}^{1 / 2}(\Omega)$, we decompose $\left\langle\mathscr{R}_{(\eta, q)}^{h}, \phi\right\rangle$ into the following two terms:

$$
\begin{aligned}
\left\langle\mathscr{R}_{(\eta, q)}^{h}, \phi\right\rangle= & \sum_{\left(x_{j}, t_{n}\right) \in \Omega} \phi_{j}^{n} \int_{x_{j-1 / 2}}^{x_{j+1 / 2}}\left(\eta\left(v_{+}^{n}\right)-\eta\left(v_{j}^{n}\right)\right) d x \\
& +\sum_{\left(x_{j}, t_{n}\right) \in \Omega} \int_{x_{j-1 / 2}}^{x_{j+1 / 2}}\left(\eta\left(v_{+}^{n}\right)-\eta\left(v_{j}^{n}\right)\right)\left(\phi-\phi_{j}^{n}\right) d x \\
\equiv & I_{1}+I_{2} .
\end{aligned}
$$

The first term can be directly estimated from Lemma 4.4,

$$
\left|I_{1}\right| \leq\|\phi\|_{C_{0}(\Omega)} \sum_{\left(x_{j}, t_{n}\right) \in \Omega}\left|\int_{x_{j-1 / 2}}^{x_{j+1 / 2}}\left(\eta\left(v_{+}^{n}\right)-\eta\left(v_{j}^{n}\right)\right) d x\right| \leq C h^{1-2 \alpha}\|\phi\|_{C_{0}(\Omega)} .
$$

Applying the Hölder inequality to the second term, we obtain

$$
\begin{aligned}
\left|I_{2}\right| \leq\left\{\sum_{\left(x_{j}, t_{n}\right) \in \Omega} \int_{x_{j-1 / 2}}^{x_{j+1 / 2}}\left|\phi\left(x, t_{n}\right)-\phi_{j}^{n}\right|^{2} d x\right\}^{\frac{1}{2}} \\
\\
\cdot\left\{\sum_{\left(x_{j}, t_{n}\right) \in \Omega} \int_{x_{j-1 / 2}}^{x_{j+1 / 2}}\left|\eta\left(v_{+}^{n}\right)-\eta\left(v_{j}^{n}\right)\right|^{2} d x\right\}^{\frac{1}{2}} .
\end{aligned}
$$

Therefore,

$$
\begin{aligned}
& \sum_{\left(x_{j}, t_{n}\right) \in \Omega} \int_{x_{j-1 / 2}}^{x_{j+1 / 2}}\left|\phi\left(x, t_{n}\right)-\phi_{j}^{n}\right|^{2} d x \\
& \quad \leq\|\phi\|_{C_{0}^{1 / 2}(\Omega)}^{2} \sum_{\left(x_{j}, t_{n}\right) \in \Omega} \int_{x_{j-1 / 2}}^{x_{j+1 / 2}}\left|x-x_{j}\right| d x \leq C\|\phi\|_{C_{0}^{1 / 2}(\Omega)}^{2},
\end{aligned}
$$

and

$$
\left|\eta\left(v_{+}^{n}\right)-\eta\left(v_{j}^{n}\right)\right| \leq\|\nabla \eta\|_{L^{\infty}}\left|v_{+}^{n}-v_{j}^{n}\right| \leq C\left|v_{+}^{n}-v_{j}^{n}\right| .
$$


Inserting (4.25) and (4.26) into (4.24), and using Lemma 4.4, yields

$$
\begin{aligned}
\left|I_{2}\right| \leq & C\|\phi\|_{C_{0}^{1 / 2}(\Omega)}\left\{\sum_{\left(x_{j}, t_{n}\right) \in \Omega} \int_{x_{j-1 / 2}}^{x_{j+1 / 2}}\left|v_{+}^{n}-v_{j}^{n}\right|^{2} d x\right\}^{\frac{1}{2}} \\
& \leq C h^{1 / 2-\alpha}\|\phi\|_{C_{0}^{1 / 2}(\Omega)}
\end{aligned}
$$

The estimates (4.22), (4.23), and (4.27) imply that

$$
\left|\left\langle\mathscr{R}_{(\eta, q)}^{h}, \phi\right\rangle\right| \leq C h^{1-2 \alpha}\|\phi\|_{C_{0}(\Omega)}+C h^{1 / 2-\alpha}\|\phi\|_{C_{0}^{1 / 2}(\Omega)} \leq C h^{1 / 2-\alpha}\|\phi\|_{C_{0}^{1 / 2}(\Omega)},
$$

or

$$
\left.\left\|\mathscr{R}_{(\eta, q)}^{h}\right\|_{\left(C_{0}^{1 / 2}(\Omega)\right.}\right)^{*} \leq C h^{1 / 2-\alpha} .
$$

This completes the proof of the lemma.

The following lemmas estimate the dissipation measures resulting from the averaging process. Their proofs are similar to $[1,6]$.

Lemma 4.6. Let $v_{h}$ be the approximate solutions of the scheme (1.5), $\eta_{*}$ be the mechanical energy, and let $\Omega \subset R_{+}^{2}$ be a bounded open set. Then we have

$$
\begin{aligned}
& M_{1} \sum_{\left(x_{j}, t_{n}\right) \in \Omega} \int_{x_{j-1 / 2}}^{x_{j+1 / 2}}\left|v_{-}^{n}-v_{j}^{n}\right|^{2} d x \\
& \quad \leq \sum_{\left(x_{j}, t_{n}\right) \in \Omega} \int_{x_{j-1 / 2}}^{x_{j+1 / 2}}\left(\eta_{*}\left(v_{-}^{n}\right)-\eta_{*}\left(v_{j}^{n}\right)\right) d x \leq M_{2},
\end{aligned}
$$

where $M_{1}$ and $M_{2}$ are positive constants independent of $h$.

Lemma 4.7. Let $\mathscr{A}_{(\eta, q)}^{h}$ be the dissipation measure resulting from the averaging process, and let $\Omega \subset R_{+}^{2}$ be a bounded open set. Then there exists a constant $C>0$ such that

(1) for any weak entropy pair $(\eta, q)$, we have

$$
\left\|\mathscr{A}_{(\eta, q)}^{h}\right\|_{\left(C_{0}^{1 / 2}(\Omega)\right)^{*}} \leq C
$$

(2) for any convex weak entropy pair $(\eta, q)$ and any $\phi \in C_{0}^{1}(\Omega), \phi \geq 0$, we have

$$
\left\langle\mathscr{A}_{(\eta, q)}^{h}, \phi\right\rangle \leq C h^{1 / 2}\|\phi\|_{C_{0}^{1}(\Omega)}
$$

(3) for the entropy pair $(v, f(v))$, we have

$$
\left\|\mathscr{A}_{(v, f(v))}^{h}\right\|_{\left(C_{0}^{1}(\Omega)\right)^{*}} \leq C h^{1 / 2} .
$$

Now, we turn to the compactness analysis of the dissipation measures $\mu_{(\eta, q)}^{h}$. We first state the following two lemmas which can be found in [1] and [15], respectively. 
Lemma 4.8. Let $\Omega \subset R^{n}$ be a bounded open set. Then

$$
\begin{aligned}
& \text { (compact set of } \left.W^{-1, q}(\Omega)\right) \cap\left(\text { bounded set of } W^{-1, r}(\Omega)\right) \\
& \subset\left(\text { compact set of } H_{\mathrm{loc}}^{-1}(\Omega)\right),
\end{aligned}
$$

where $q$ and $r$ are constants, $1<q \leq 2<r<\infty$.

Lemma 4.9. The embedding of the positive cone of $W^{-1, p}$ in $W^{-1, q}$ is completely continuous for all $q<p$.

Theorem 4.1. Let $\mu_{(\eta, q)}^{h}$ be the entropy dissipation measures associated with the approximate solutions $v_{h}=\left(\rho_{h}, m_{h}\right)$ of the scheme (1.5). Suppose the initial data $v_{0}=\left(\rho_{0}(x), m_{0}(x)\right) \in \Sigma_{M}$ for some constant $M>0$. Then, for any weak entropy pair $(\eta, q)$, we have

$$
\mu_{(\eta, q)}^{h} \quad \text { is compact in } H_{\mathrm{loc}}^{-1}\left(R_{+}^{2}\right) \text {. }
$$

Proof. Step 1. Let $\Omega \subset R_{+}^{2}$ be any bounded open set. From the uniform boundedness of $v_{h}$, we have

$$
\mu_{(\eta, q)}^{h} \quad \text { is bounded in } W^{-1, \infty}(\Omega) .
$$

Since $\Omega$ is bounded, this statement implies

$$
\mu_{(\eta, q)}^{h} \quad \text { is bounded in } W^{-1, r}(\Omega), r>1 .
$$

We have from Lemma 4.5 and Lemma 4.7 that

$$
\mathscr{R}_{(\eta, q)}^{h}+\mathscr{A}_{(\eta, q)}^{h} \quad \text { is bounded in }\left(C_{0}^{1 / 2}(\Omega)\right)^{*} .
$$

The Sobolev embedding theorem gives

$$
\left(C_{0}^{1 / 2}(\Omega)\right)^{*} \hookrightarrow W_{0}^{-1,4 / 3}(\Omega),
$$

and, for any $1<p<4 / 3$,

$$
\left(C_{0}^{1 / 2}(\Omega)\right)^{*} \stackrel{\text { compact }}{\hookrightarrow} W_{0}^{-1, p}(\Omega) .
$$

From (4.34)-(4.35), we get

$$
\mathscr{R}_{(\eta, q)}^{h}+\mathscr{A}_{(\eta, q)}^{h} \quad \text { is bounded in } W^{-1,4 / 3}(\Omega),
$$

and, for any $1<q<4 / 3$,

$$
\mathscr{R}_{(\eta, q)}^{h}+\mathscr{A}_{(\eta, q)}^{h} \quad \text { is compact in } W^{-1, q}(\Omega) \text {. }
$$

Step 2. For any convex weak entropy pair $(\eta, q)$, we have from $(4.13)-(4.14)$ that

$$
\mu_{(\eta, q)}^{h}-\mathscr{R}_{(\eta, q)}^{h}-\mathscr{A}_{(\eta, q)}^{h} \leq 0 .
$$

We obtain from (4.33) and (4.36) that

$$
\left\|\mu_{(\eta, q)}^{h}-\mathscr{R}_{(\eta, q)}^{h}-\mathscr{A}_{(\eta, q)}^{h}\right\|_{W^{-1,4 / 3}} \leq C .
$$

We conclude from (4.38)-(4.39) and Lemma 4.9 that, for any $1<q<4 / 3$,

$$
\mu_{(\eta, q)}^{h}-\mathscr{R}_{(\eta, q)}^{h}-\mathscr{A}_{(\eta, q)}^{h} \quad \text { is compact in } W^{-1, q}(\Omega) .
$$


Thus (4.37) and (4.40) imply that, for any $1<q<4 / 3$,

$$
\mu_{(\eta, q)}^{h} \quad \text { is compact in } W^{-1, q}(\Omega) .
$$

By Lemma 4.8, (4.33), (4.41), and the arbitrariness of $\Omega$, we have

$$
\mu_{(\eta, q)}^{h} \quad \text { is compact in } H_{\text {loc }}^{-1}\left(R_{+}^{2}\right) .
$$

Step 3. For any weak convex entropy pair $(\eta, q)$, we know from Lemma 4.3 that, for any constant $K>0$, there is a constant $C>0$ such that

$$
\left|\nabla^{2} \eta(v)\right| \leq C \nabla^{2} \eta_{*}(v), \quad \forall v \in \Sigma_{K} .
$$

Following an idea of Chen [3], we construct another weak entropy pair

$$
(\bar{\eta}, \bar{q})=C\left(\eta_{*}, q_{*}\right)-(\eta, q) \text {. }
$$

We note from (4.43) that $(\bar{\eta}, \bar{q})$ is a convex weak entropy pair on the set $\Lambda_{C_{0}}$. By the definition of the dissipation measures (2.5), we have

$$
\mu_{(\eta, q)}^{h}=C \mu_{\left(\eta_{*}, q_{*}\right)}^{h}-\mu_{(\eta, q)}^{h} .
$$

Applying (4.42) to the convex entropies $\eta_{*}$ and $\bar{\eta}$, we obtain

$$
\mu_{(\eta, q)}^{h} \quad \text { is compact in } H_{\mathrm{loc}}^{-1}\left(R_{+}^{2}\right) .
$$

This completes the proof of the theorem.

4.3. Convergence analysis. Now we establish the convergence theorem and prove that the limit function satisfies the entropy inequalities.

Theorem 4.3. Suppose that the initial data $\left(\rho_{0}(x), m_{0}(x)\right)$ satisfy

$$
0 \leq \rho_{0}(x) \leq M, \quad\left|m_{0}(x)\right| \leq M \rho_{0}(x),
$$

for a constant $M>0$. Then the approximate solution sequence $v_{h}=\left(\rho_{h}, m_{h}\right)$ of the scheme (1.5) has a convergent subsequence (still labeled) $v_{h}(x, t)$ such that

$$
\left(\rho_{h}(x, t), m_{h}(x, t)\right) \rightarrow(\rho(x, t), m(x, t)), \quad \text { a.e. },
$$

and the function pair $(\rho(x, t), m(x, t))$ is a generalized solution of the Cauchy problem (1.1)-(1.2). Furthermore, there is a constant $M_{T}>0$ such that

$$
0 \leq \rho(x, t) \leq M_{T}, \quad|m(x, t)| \leq M_{T} \rho(x, t), \quad \text { a.e. },
$$

for any $x \in R, 0 \leq t \leq T$.

The proof of this theorem is similar to that of $[1,6]$, using Lemma 4.4 and Lemma 4.5. We leave it to the reader.

\section{EXTENSION TO INITIAL BOUNDARY PROBLEMS}

In this section we extend our results to the following "double piston problem", which is the simplest case of the mixed initial boundary value problem for the system of isentropic gas dynamics and has many physical applications:

$$
\left\{\begin{array}{l}
\rho_{t}+m_{x}=0, \quad(x, t) \in D, \\
m_{t}+\left(m^{2} / \rho+p(\rho)\right)_{x}=0, \quad(x, t) \in D, \\
\left.(\rho, m)\right|_{t=0}=\left(\rho_{0}(x), m_{0}(x)\right), \quad 0<x<L, \\
m\left(x_{j}(t), t\right)-\rho\left(x_{j}(t), t\right) u_{j}(t)=0, \quad t>0, \quad j=1,2,
\end{array}\right.
$$


where $\rho$ is the density, $m$ is the momentum, $p(\rho)=\rho^{\gamma} / \gamma$ is the pressure, $1<\gamma \leq 5 / 3$ is the adiabatic exponent, $u_{1}(t)$ and $u_{2}(t)$ are the velocities of the pistons, $x_{1}(t)$ and $x_{2}(t)$ are the moving boundaries, and

$$
\left\{\begin{array}{l}
D=\left\{(x, t): x_{1}(t)<x<x_{2}(t), t>0\right\} \\
x_{1}(t)=\int_{0}^{t} u_{1}(s) d s, \quad x_{2}(t)=L+\int_{0}^{t} u_{2}(s) d s .
\end{array}\right.
$$

"Two interacting blast waves", a special case of the double piston problem with $u_{1}(t)=u_{2}(t)=0$ and some specific initial data, has become the standard numerical test problem for numerical schemes [27].

Definition 5.1. A pair of bounded measurable functions $(\rho(x, t), m(x, t))$ is called a generalized solution of the mixed problem (5.1) in the region $D$ if it satisfies the following conditions:

(1) For any $\phi(x, t), \psi(x, t) \in C_{0}^{\infty}\left(R_{+}^{2}\right),(\phi, \psi)\left(x_{j}(t), t\right) \equiv 0, j=1,2$, we have

$$
\left\{\begin{array}{l}
\iint_{D}\left(\rho \phi_{t}+m \phi_{x}\right) d x d t+\int_{-\infty}^{\infty} \rho_{0}(x) \phi(x, 0) d x=0 \\
\iint_{D}\left(m \psi_{t}+\left(\rho m^{2} / \rho+p(\rho)\right) \psi_{x}\right) d x d t+\int_{-\infty}^{\infty} m_{0}(x) \psi(x, 0) d x=0
\end{array}\right.
$$

(2) For any convex weak entropy pair $(\eta, q)$ and any function $\phi(x, t) \in$ $C_{0}^{\infty}\left(R_{+}^{2}\right)$ satisfying

$$
\phi(x, t) \geq 0, \quad \phi(x, 0) \equiv 0, \quad \phi\left(x_{j}(t), t\right) \equiv 0, \quad j=1,2,
$$

we have

$$
\iint_{D}\left(\eta(v) \phi_{t}+f(v) \phi_{x}\right) d x d t \geq 0
$$

The construction of the generalized solution for the mixed problem (5.1) with the following generalized Riemann data can be found in [16]:

$$
\left\{\begin{array}{l}
\left.(\rho, m)\right|_{t=0}=\left(\rho_{r}, m_{r}\right), \quad \text { for } x>0, \\
m\left(u_{0} t, t\right)-u_{0} \rho\left(u_{0} t, t\right)=0, \quad \text { for } t>0,
\end{array}\right.
$$

where $u_{0}, \rho_{r}, m_{r}$ are three constants. Once we have these generalized Riemann solutions, we can construct the Godunov scheme and prove the existence of the generalized solution for the mixed problem. The following theorem is given by Takeno [22].

Theorem 5.1. There is a global generalized solution for the mixed initialboundary value problem (5.1).

By the properties of the solutions of the Riemann problems and the generalized Riemann problems, and the convergence of the Godunov scheme, we have the following properties for the generalized solution (constructed by the Godunov scheme) of the mixed problems (5.1). 
Lemma 5.2. For the generalized solution constructed by the Godunov scheme, for any $t_{1}, t_{2} \in[0, T]$, any convex weak entropy $(\eta, q)$, and any $\phi \in C_{0}^{\infty}\left(R_{+}^{2}\right)$, $\phi \geq 0, \phi\left(x_{j}(t), t\right)=0, j=1,2$, the solution $v(x, t)$ satisfies

$$
\begin{aligned}
& \int_{t_{1}}^{t_{2}} \int_{x_{1}(t)}^{x_{2}(t)}\left(\eta(v) \phi_{t}+q(v) \phi_{x}\right) d x d t \\
& \quad \geq \int_{x_{1}\left(t_{1}\right)}^{x_{2}\left(t_{1}\right)} \eta\left(v\left(x, t_{1}\right)\right) \phi\left(x, t_{1}\right) d x-\int_{x_{1}\left(t_{2}\right)}^{x_{2}\left(t_{2}\right)} \eta\left(v\left(x, t_{2}\right)\right) \phi\left(x, t_{2}\right) d x .
\end{aligned}
$$

Lemma 5.3. Suppose that the Riemann invariants $\left(w_{0}(x), z_{0}(x)\right)$ of the initial data $\left(\rho_{0}(x), m_{0}(x)\right)$ satisfy

$$
z_{0}(x) \leq u_{1}(t) \leq \frac{1}{2}\left(w_{0}(x)+z_{0}(x)\right), \quad \frac{1}{2}\left(w_{0}(x)+z_{0}(x)\right) \leq u_{2}(t) \leq w_{0}(x) .
$$

Then the regions $\Lambda_{C}, C>0$, are the invariant regions for mixed problems (5.1). More precisely, if the initial data belong to $\Lambda_{C}$, then the generalized solutions constructed by the Godunov scheme for the mixed problem (5.1) also belong to $\Lambda_{C}$.

As in the construction of the approximate solutions of the scheme (1.5) for the Cauchy problem (1.1)-(1.2) in $\S 3$, we denote $h$ and $\Delta t$ as the space step length and the time step length, respectively. We partition the real line of the space variable into cells, with the $j$ th cell centered at $x_{j}=j h, j=$ $0, \pm 1, \pm 2, \ldots$. We denote $\left\{j_{n}, J_{n}\right\}$ as a sequence of integers such that the moving boundaries $x_{1}(t)$ and $x_{2}(t)$ at the time $t_{n}$ satisfy

$$
x_{j_{n}-1 / 2} \leq x_{1}\left(t_{n}\right)<x_{j_{n}+1 / 2}, \quad x_{J_{n}-1 / 2} \leq x_{2}\left(t_{n}\right)<x_{J_{n}+1 / 2} .
$$

We define internal cells, for $n=0,1, \ldots$, and $j=j_{n}+2, j_{n}+3, \ldots, J_{n}-2$,

$$
I_{j}^{n}=\left[x_{j-1 / 2}, x_{j+1 / 2}\right) \times\left[t_{n}, t_{n+1}\right),
$$

and boundary cells, for $n=0,1, \ldots$,

$$
\left\{\begin{array}{l}
B_{1}^{n}=\left\{(x, t), \quad x_{1}(t) \leq x<x_{j_{n}+3 / 2}, \quad t_{n} \leq t<t_{n+1}\right\}, \\
B_{2}^{n}=\left\{(x, t), \quad x_{J_{n}-3 / 2} \leq x<x_{2}(t), \quad t_{n} \leq t<t_{n+1}\right\} .
\end{array}\right.
$$

We let $\Delta t / h$ be small enough such that

$$
x_{1}\left(t_{n+1}\right)<x_{j_{n}+3 / 2}, \quad x_{2}\left(t_{n+1}\right)>x_{J_{n}-3 / 2} .
$$

As in the standard MUSCL scheme, there are three steps: reconstruction, solution in small time, and cell averaging. The reconstruction and the cell averaging steps are the same as for the Cauchy problem (see $\S 3$ ). In the "solution in small time" step, we also have the generalized solution for both internal and boundary cells as a result of the convergence of the Godunov scheme for mixed problems.

We can also use the following linear interpolation $x_{1}^{h}(t)$ to approximate $x_{1}(t)$ in the MUSCL scheme. On the intervals $t_{n}<t \leq t_{n+1}, \quad n=0,1,2, \ldots$, we define

$$
x_{1}^{h}(t)=x_{1}^{h}\left(t_{n}\right)+\frac{t-t_{n}}{\Delta t} \int_{t_{n}}^{t_{n+1}} u_{1}(t) d t,
$$

with $x_{1}^{h}(0)=0$. 
Theorem 5.2. Let $v_{h}(x, t)=\left(\rho_{h}(x, t), m_{h}(x, t)\right)$ be the approximate solutions of the above scheme for the mixed initial boundary value problem (5.1)-(5.2). Suppose that the initial data $\left(\rho_{0}(x), m_{0}(x)\right)$ satisfy

$$
0 \leq \rho_{0}(x) \leq M, \quad\left|m_{0}(x)\right| \leq M \rho_{0}(x),
$$

for some constants $M>0$, and the moving boundaries $x_{1}(t)$ and $x_{2}(t)$ satisfy

$$
x_{2}(t)-x_{1}(t) \geq \delta(t), \quad \text { for } t>0,
$$

where $\delta(t)$ is some positive continuous function. Then there exists a convergent subsequence (still labeled) $v_{h}(x, t)$ such that

$$
\left(\rho_{h}(x, t), m_{h}(x, t)\right) \rightarrow(\rho(x, t), m(x, t)), \quad \text { a.e. }(x, t) \in D
$$

and the function pair $(\rho(x, t), m(x, t))$ is a generalized solution of the mixed problem (5.1)-(5.2).

The proof of this theorem is similar to that of the Cauchy problem (see $\S 4$ ) except that we use Lemma 5.2 and Lemma 5.3 instead of Theorem 2.2 and Lemma 4.2. We estimate the boundary cells and the internal cells separately.

\section{BIBLIOGRAPHY}

1. G.-Q. Chen, The compensated compactness method and the system of isentropic gas dynamics, MSRI preprint 00527-91, 1990.

2. Convergence of the Lax-Friedrichs scheme for isentropic gas dynamics III, Acta Math. Sci. 6 (1986), 75-120; 8 (1988), 243-276 (in Chinese).

3. _ Hyperbolic systems of conservation laws with a symmetry, Comm. Partial Differential Equations 16 (1991), 1461-1487.

4. G.-Q. Chen, Q. Du, and E. Tadmor, Spectral viscosity approximations to multidimensional scalar conservation laws, Math. Comp. 61 (1993), 629-643.

5. F. Coquel and P. Le Floch, Convergence of finite difference schemes for conservation laws in several space dimensions: The corrected antidiffusive flux approach, Math. Comp. 57 (1991), 169-210.

6. X. Ding, G.-Q. Chen, and P. Luo, Convergence of the Lax-Friedrichs scheme for isentropic gas dynamics I, II, Acta Math. Sci. 5 (1985), 483-500, 501-540; 7 (1987), 460-480, 8 (1988), 61-94 (in Chinese).

7. R. DiPerna, Convergence of viscosity method for isentropic gas dynamics, Comm. Math. Phys. 91 (1983), 1-30.

8. S. K. Godunov, A finite difference scheme for the numerical computation of discontinuous solutions of the equations of fluid dynamics, Mat. Sb. 47 (1959), 271-290.

9. A. Harten and S. Osher, Uniformly high order accurate non-oscillatory schemes, I, SIAM J. Numer. Anal. 24 (1987), 279-309.

10. A. Harten, B. Engquist, S. Osher, and S. Chakravarthy, Uniformly high order accurate non-oscillatory schemes, III, J. Comput. Phys. 71 (1987), 231-303.

11. P. D. Lax, Hyperbolic systems of conservation laws and the mathematical theory of shock waves, SIAM, Philadelphia, PA, 1973.

12. P. D. Lax and B. Wendroff, Systems of conservation laws, Comm. Pure Appl. Math. 13 (1960), 217-237.

13. P. L. Lions and P. Souganidis, Convergence of MUSCL type methods for scalar conservation laws, C. R. Acad. Sci. Paris, Série I 311 (1990), 259-264.

14. A. Majda and S. Osher, Numerical viscosity and the entropy condition, Comm. Pure Appl. Math. 32 (1979), 797-838. 
15. F. Murat, L'injection du cone positif de $H^{-1}$ dans $W^{-1, q}$ est compacte pour tout $q<2$, J. Math. Pure Appl. 60 (1981), 309-322.

16. T. Nishida and J. Smoller, Mixed problems for nonlinear conservation laws, J. Differential Equations 23 (1977), 244-269.

17. S. Osher, On convergence of generalized MUSCL schemes, SIAM J. Numer. Anal. 22 (1985), 947-961.

18. P. L. Roe, Approximate Riemann solvers, parameter vectors, and difference schemes, J. Comput. Phys. 43 (1981), 357-380.

19. C.-W. Shu and S. Osher, Efficient implementation of essentially non-oscillatory shock capturing schemes, J. Comput. Phys. 83 (1989), 32-51.

20. A. Szepessy, Convergence of a shock-capturing streamline diffusion finite element method for a scalar conservation law in two space dimensions, Math. Comp. 53 (1989), 527-545.

21. E. Tadmor, Semi-discrete approximations to nonlinear systems of conservation laws: consistency and $L^{\infty}$-stability imply convergence, ICASE report 88-41, 1988.

22. S. Takeno, Initial boundary problems for isentropic gas dynamics, Proc. Roy. Soc. Edinburgh 120A (1992), 1-23.

23. L. Tartar, Compensated compactness and applications to partial differential equations, Research Notes in Math., Nonlinear Analysis and Mechanics (R. J. Knops, ed.), vol. 4, Pitman Press, New York, 1979.

24. B. Van Leer, Towards the ultimate conservative difference schemes, V, A second order sequel to Godunov's method, J. Comput. Phys. 43 (1981), 357-372.

25. J. P. Vila, High-order schemes and entropy condition for nonlinear hyperbolic systems of conservation laws, Math. Comp. 50 (1988), 53-73.

26. J. von Neumann and R. D. Richtmyer, A method for the numerical calculation of hydrodynamic shocks, J. Appl. Phys. 21 (1950), 232-237.

27. P. Woodward and P. Colella, The numerical simulation of two-dimensional fluid flow with strong shock, J. Comput. Phys. 54 (1984), 115-173.

28. H. Yang, Nonlinear wave analysis and convergence of MUSCL schemes, IMA preprint 697, 1990.

Department of Mathematics, The University of Chicago, Chicago, Illinois 60637

E-mail address: cheng@math.uchicago.edu

Courant Institute of Mathematical Sciences, New York, New York 10012

E-mail address: jliu@cims20.nyu.edu 